ARTICLE

DOI: $10.1038 / s 41467-018-05701-8$

\title{
Engineering the spin couplings in atomically crafted spin chains on an elemental superconductor
}

\author{
A. Kamlapure (10 ${ }^{1}$, L. Cornils ${ }^{1}$, J. Wiebe (i] ${ }^{1} \&$ R. Wiesendanger ${ }^{1}$
}

Magnetic atoms on a superconductor give rise to Yu-Shiba-Rusinov (YSR) states within the superconducting energy gap. A spin chain of magnetic adatoms on an s-wave superconductor may lead to topological superconductivity accompanied by the emergence of Majorana modes at the chain ends. For their usage in quantum computation, it is a prerequisite to artificially assemble the chains and control the exchange couplings between the spins in the chain and in the substrate. Here, using a scanning tunneling microscope tip, we demonstrate engineering of the energy levels of the YSR states by placing interstitial Fe atoms in close proximity to adsorbed Fe atoms on an oxidized Ta surface. Based on this prototype platform, we show that the interaction within a long chain can be strengthened by linking the adsorbed Fe atoms with the interstitial ones. Our work adds an important step towards the controlled design and manipulation of Majorana end states.

\footnotetext{
${ }^{1}$ Department of Physics, University of Hamburg, Jungiusstrasse 9-11, D-20355 Hamburg, Germany. Correspondence and requests for materials should be addressed to A.K. (email: akamlapu@physnet.uni-hamburg.de)
} 
M ajorana particles ${ }^{1}$ have been proposed as key elements for topological quantum computation ${ }^{2-5}$ due to their unique statistical properties. One of the systems for realizing Majorana modes in condensed matter is a spin chain on a superconductor ${ }^{6-9}$. The recent investigation of self-assembled ferromagnetic $\mathrm{Fe}$ chains on superconducting $\mathrm{Pb}$, featuring strong spin-orbit coupling, triggered enhanced interest in the possible realization of Majorana modes at the ends of such chains ${ }^{10-15}$. So far, this platform for Majorana physics has been tested almost exclusively for the substrate material $\mathrm{Pb}$ hampering the controlled assembly of magnetic atoms into chains or more complex networks, which are ultimately needed for braiding of Majorana modes and their usage in fault-tolerant quantum computation ${ }^{3-5}$. Moreover, the required Yu-Shiba-Rusinov (YSR) ${ }^{16-18}$ band formation crucially depends on the exchange couplings between the spins within the chain ${ }^{19-21}$ and between the spins and the conduction electrons of the host material ${ }^{22,23}$. Therefore, it is desirable to artificially construct such spin chains with full control over all the couplings in order to drive the system into desired topological phases ${ }^{24}$.

To this end, we explore the superconducting substrate of a $(3 \times$ 3) oxygen reconstructed $\mathrm{Ta}(100)$ surface (named $\mathrm{Ta}(100)-(3 \times 3) \mathrm{O}$ in the following) decorated with $\mathrm{Fe}$ atoms $\mathrm{s}^{22}$. We first show that a spin chain constructed on the bare oxidized $\mathrm{Ta}(100)$ surface shows negligible interaction between nearest-neighbor $\mathrm{Fe}$ atoms. In the second step, we introduce $\mathrm{Fe}$ atoms in the interstitial sites in close proximity to the $\mathrm{Fe}$ adatoms using the tip of a scanning tunneling microscope (STM) and demonstrate the control over the energy levels of the YSR states. Finally we extend the method to build long chains of $\mathrm{Fe}$ adatoms that are interacting via interstitial $\mathrm{Fe}$ atoms and demonstrate spin coupling within the chain.

\section{Results}

Assembly and investigation of chains of Fe adatoms. As seen in the topographic STM image of the substrate (Fig. 1a), the regular network of oxygen atoms is imaged as a network of depression lines separating circular and cross-shaped plaquettes of $(3 \times 3) \mathrm{Ta}$ atoms ${ }^{22}$. After low-temperature Fe deposition we see a statistical distribution of $\mathrm{Fe}$ adatoms with different apparent heights showing different spectroscopic features. In particular, one type of adatoms reveals a YSR state at a binding energy which varies for adsorption on a locally different substrate environment ${ }^{22}$. We were able to use vertical atom manipulation ${ }^{25,26}$ to construct arrays of $\mathrm{Fe}$ adatoms positioned on the centers of neighboring cross-shaped plaquettes (Fig. 1b-d, f; see Methods Section). Figure 1b-d show a single manipulated $\mathrm{Fe}$ adatom, a manipulated pair and a manipulated three-atom chain. Differential tunneling conductance $(\mathrm{d} I / \mathrm{d} V)$ spectra taken with a superconducting tip on the manipulated atoms of the three structures (Fig. 1e) show a pair of peaks at energies close to the gap edge indicative of a YSR state with a relatively large binding energy $\left(E_{\mathrm{b}} \sim \Delta\right)^{22}$. Note, that the YSR state neither does change with the number of atoms in the chain nor with the number of neighbors, indicating negligible interaction amongst the atoms on neighboring plaquettes ${ }^{16,21}$. This holds for a long chain built from 63 atoms (Fig. 1f, Supplementary Fig. 1) where we see similar spectroscopic features along the chain as well as at the ends.

In order to verify the negligible coupling between neighboring $\mathrm{Fe}$ atoms in the chains, we used the following procedure. Applying a small bias pulse (typically $\pm 500 \mathrm{mV}$ ) to a manipulated Fe adatom, its electronic configuration can be switched between two states with strikingly different spectroscopic signature (Fig. 2a-d). For the original case discussed above (Fig. 2a), the spectrum shows the YSR states in the gap (Fig. 2c) and a resonance with a full width at half maximum on the order of $50 \mathrm{mV}$, indicating a partial Kondo screening of the magnetic moment of the $\mathrm{Fe}$ atom (Fig. $2 \mathrm{~d})^{23}$. In the following, we denominate this state of the Fe adatom as the "YSR-on" state. After applying a positive voltage pulse (Fig. $2 \mathrm{~b}$ ), the spectrum on the $\mathrm{Fe}$ adatom completely changed to the spectroscopic signature of a substrate spectrum (Fig. 2c, d). This most probably indicates that the $\mathrm{Fe}$ adatom has completely lost its magnetic moment. We refer to the corresponding state as the "YSR-off" state. Note, that the process is reversible by the application of a voltage pulse of reversed bias polarity. We suppose that the voltage-pulse induced switching of the electronic and magnetic properties of the $\mathrm{Fe}$
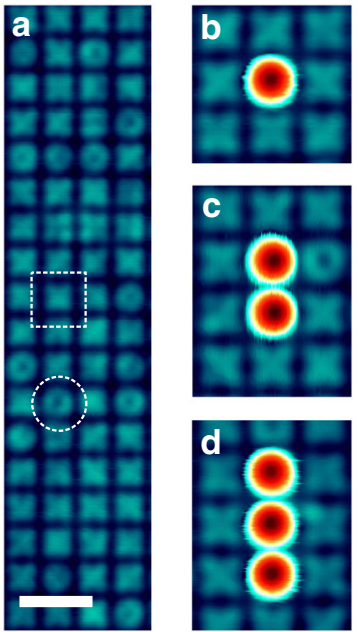

$0 \AA$
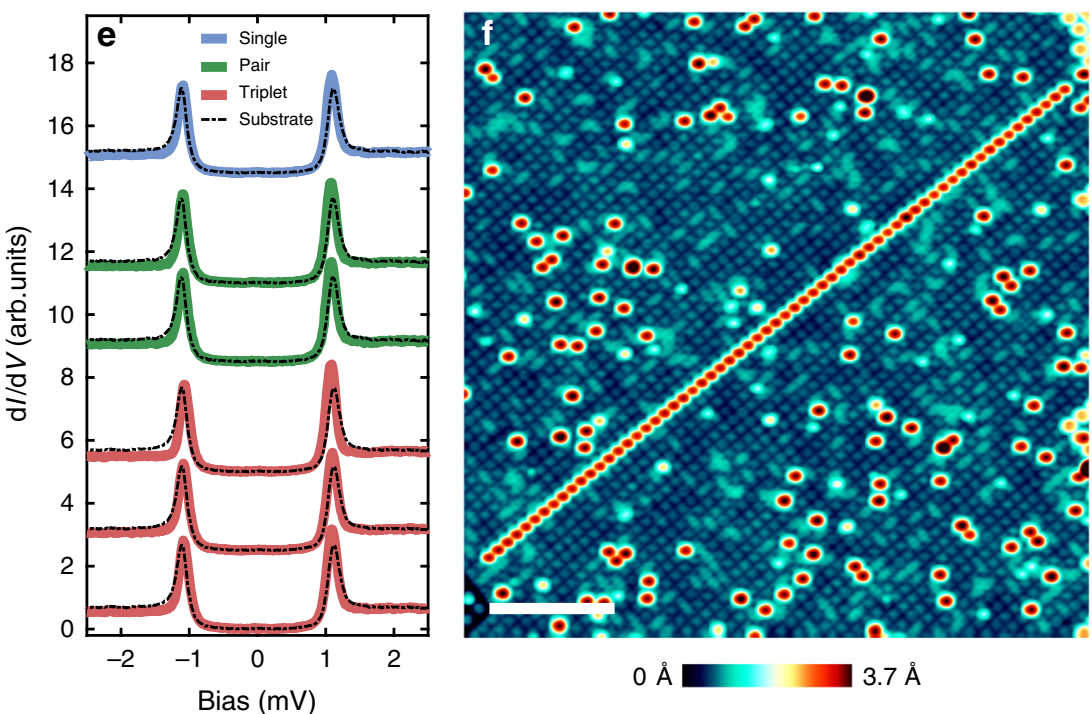

Fig. 1 Spectroscopy of short chains of Fe adatoms. a Constant-current STM image of the typical surface of Ta (100)-O showing a regular array of crossshaped (dashed rectangle) and circular shaped (dashed circle) plaquettes. White scale bar is $2 \mathrm{~nm}$. b-d STM images showing a single Fe adatom, a pair, and a three-atom chain that were assembled using atom manipulation. e dl/dV spectra measured with a superconducting tip on top of the different chains of Fe adatoms shown in b-d. Each spectrum is plotted together with the spectrum of the substrate taken with the same tip apex. Spectra are shifted vertically and grouped for clarity (STS parameters: $V_{\text {stab }}=2.5 \mathrm{mV}, I_{\text {stab }}=100 \mathrm{pA}, V_{\text {mod }}=20 \mu \mathrm{V}$, scan parameters: $\mathrm{V}=50 \mathrm{mV}, I=100 \mathrm{pA}$ ). f STM image of a long chain made of $63 \mathrm{Fe}$ adatoms. White scale bar is $10 \mathrm{~nm}$ long $(V=100 \mathrm{mV}, I=100 \mathrm{pA})$ 

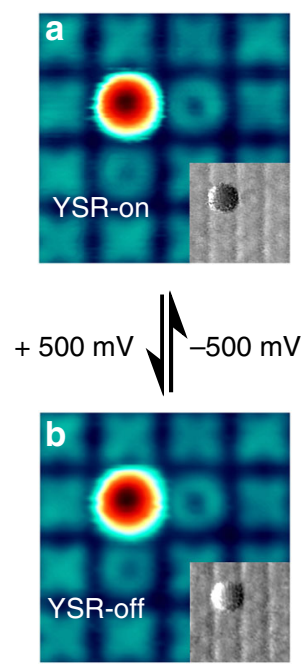

$0 \AA$
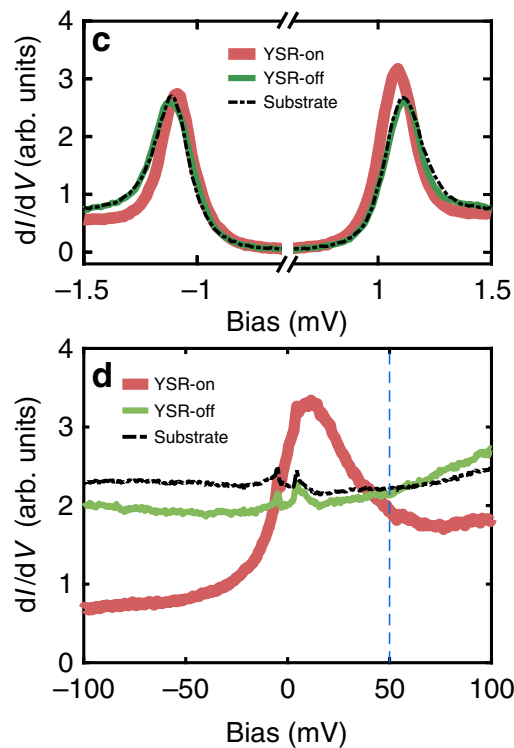
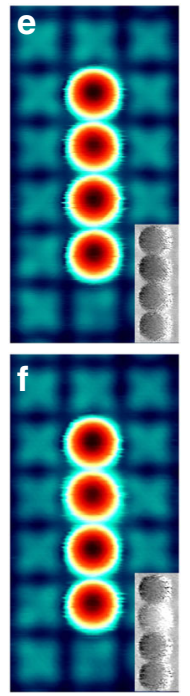

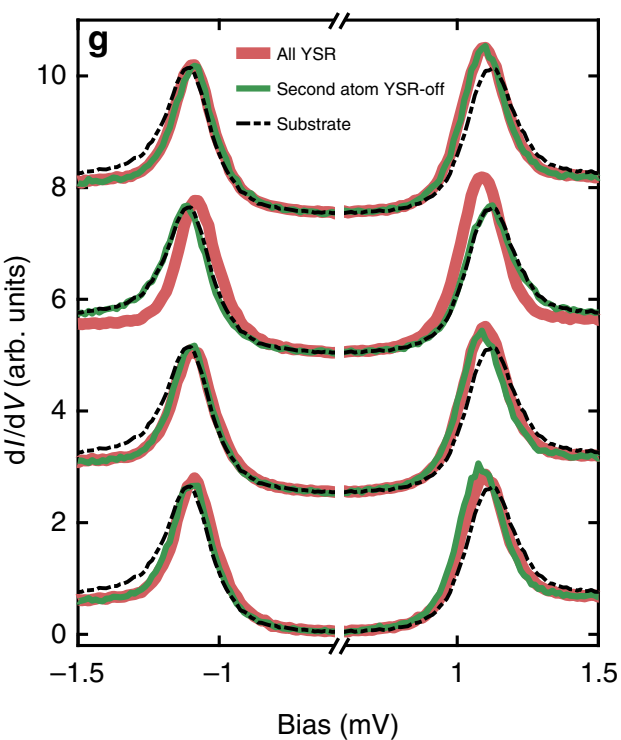

Fig. 2 Switching between YSR-on and YSR-off state in a four-atom chain. a, b STM image showing a single Fe adatom in YSR-on and YSR-off state, respectively. The typical voltage pulse required to switch between the two states is indicated between the two panels. The inset in each panel shows the $\mathrm{d} / / \mathrm{dV}$ image at $V=50 \mathrm{mV}$. c, $\mathbf{d} \mathrm{dl} / \mathrm{d} V$ spectra taken with a superconducting tip on the adatom for the two cases shown in $\mathbf{a}$, $\mathbf{b}$, plotted together with the substrate spectrum. e, f STM image of a four-atom chain with the second atom in YSR-on and YSR-off state, respectively. Insets in each panel represent dl/ $\mathrm{d} V$ images. $\mathbf{g} \mathrm{d} / / \mathrm{d} V$ spectra taken with a superconducting tip on each adatom of the four-atom chain for the two cases shown in $\mathbf{e}, \mathbf{f}$, plotted together with the substrate spectrum. The spectra are shifted vertically for each adatom for clarity. Scan parameters: $V=50 \mathrm{mV}, I=100 \mathrm{pA}, V_{\text {mod }}=4 \mathrm{mV}$. Stabilization parameters for STS in $\mathbf{c}$ and $\mathbf{g}: V_{\text {stab }}=2.5 \mathrm{mV}, I_{\text {stab }}=100 \mathrm{pA}, V_{\text {mod }}=20 \mu \mathrm{V}$ and those for $\mathbf{d}: V_{\text {stab }}=100 \mathrm{mV}, I_{\text {stab }}=100 \mathrm{pA}, V_{\text {mod }}=3 \mathrm{mV}$
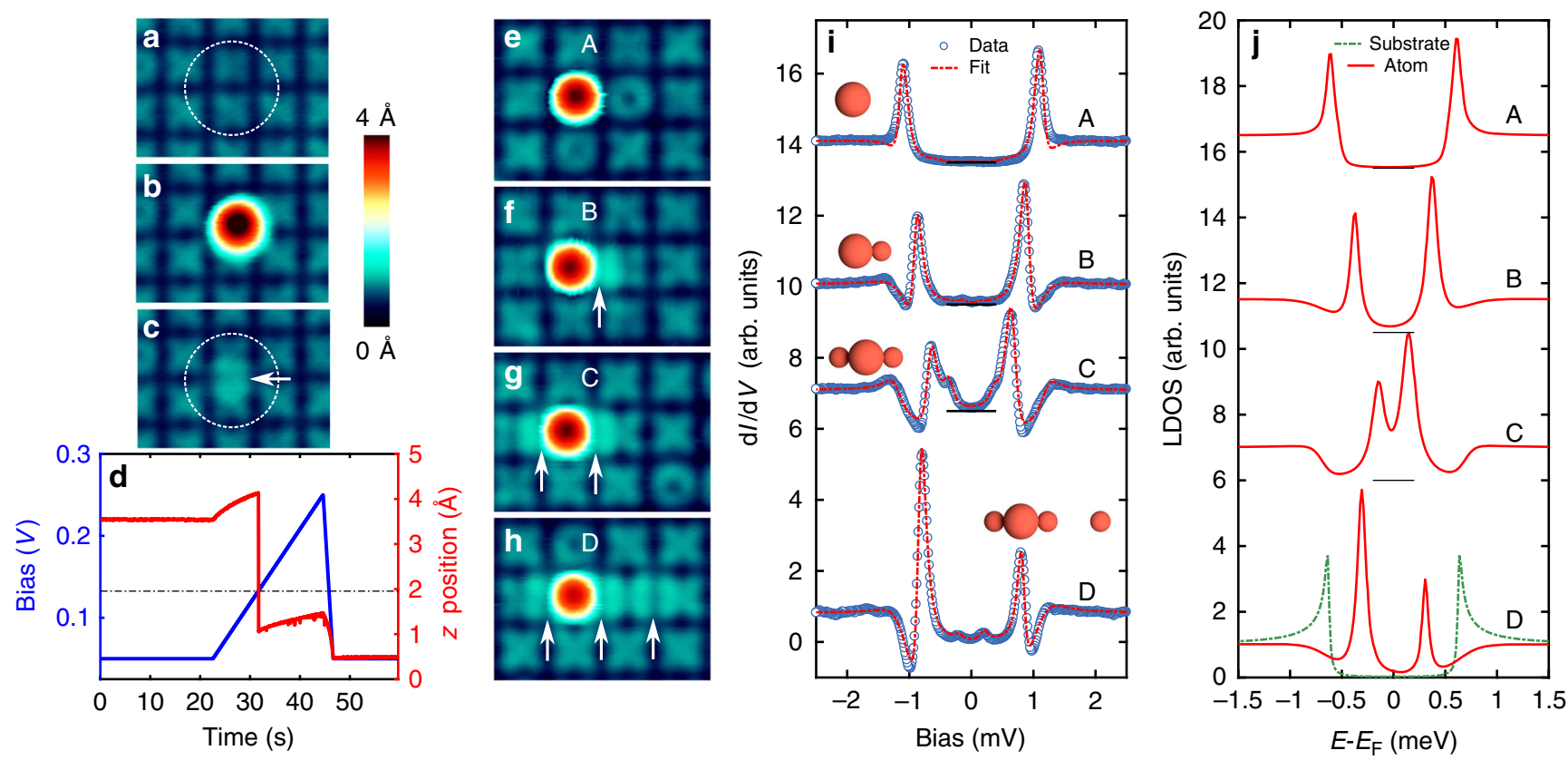

Fig. 3 Spectroscopy on adatoms coupled to interstitial Fe atoms (IFAs). a STM image of the area before the construction of the IFA. Dashed circle represents the target point. $\mathbf{b}$ STM image after vertical manipulation of an adatom to the target point. $\mathbf{c}$ Image after introducing the IFA into the target site. d Bias voltage and $z$ position of the tip as a function of time during the manipulation of the Fe from the position in $\mathbf{b}$ to the interstitial site in $\mathbf{c}$. Horizontal dashed line indicates the bias value, $V=130 \mathrm{mV}$, at which the Fe adatom relaxes into the interstitial site. e-h STM image of adatoms with no (a), one (b), two (c) and three (d) IFAs in close proximity. Here, the white arrows represent the positions of IFAs. i dl/dV spectra taken with a superconducting tip along with the fit using numerical deconvolution corresponding to the adatoms shown in e-h. Spectra are shifted vertically for clarity. The pictograms indicate the positions of adatoms (large spheres) and IFAs (small spheres). $\mathbf{j}$ Numerically deconvoluted local density of states (LDOS) corresponding to the fits in $\mathbf{i}$. Substrate density of states is plotted as reference. Stabilization parameters for STS: $V_{\text {stab }}=2.5 \mathrm{mV}, I_{\text {stab }}=100 \mathrm{pA}, V_{\text {mod }}=20 \mu V$ and scan parameters: $V=50 \mathrm{mV}, I=100 \mathrm{pA}$ 
adatom is possibly due to a switching between two different metastable states of the $\mathrm{Fe}$ atom on the $\mathrm{Ta}(100)-(3 \times 3) \mathrm{O}$ reconstruction 27,28 . Using these two different spectroscopic signatures, we can also identify the state of the corresponding Fe adatom in a $\mathrm{d} I / \mathrm{d} V$ image recorded at $V=50 \mathrm{mV}$ (insets of Fig. 2a, b), revealing a lower $\mathrm{d} I / \mathrm{d} V$ signal in the YSR-on state as compared to the YSR-off state. Below, we will investigate the effect of the controlled switching of an Fe adatom within the chains between the magnetic (YSR-on) state and the nonmagnetic (YSR-off) state on the YSR states of the neighboring atoms, which will enable us to conclude on the magnetic coupling between the atoms in the chain ${ }^{16,21}$. This is demonstrated in a four-atom chain (Fig. $2 \mathrm{e}-\mathrm{g}$ ). When an atom within the chain is switched from the YSR-on state (Fig. 2e) to the YSR-off state (Fig. 2f shown exemplarily for the second atom from the top), the $\mathrm{d} I / \mathrm{d} V$ spectra on all other atoms within the chain look almost identical (Fig. 2g). We, therefore, conclude that chains built from adatoms on neighboring plaquettes reveal negligible interatomic Fe interaction preventing the formation of a YSR band, as expected by the rather large interatomic distance $(\sim 1 \mathrm{~nm})$ with a decoupling oxygen row in-between ${ }^{22}$.

Interstitial atom manipulation and creating Fe arrays. In order to increase the coupling between the Fe adatoms, we devised a unique technique of manipulating Fe atoms into interstitial sites in the substrate in-between the Fe adatoms. Using the STM tip employing vertical manipulation (see the Methods section), an $\mathrm{Fe}$ atom is first dropped close to the target region onto an $\mathrm{O}$ row inbetween two plaquettes (Fig. 3a, b). With the tip held above this Fe atom, the bias is then slowly increased (Fig. 3d) until at a typical bias voltage between 100 and $300 \mathrm{mV}$ a sudden decrease in the $z$ position of the tip is seen. This indicates that the Fe atom moved into an interstitial site in the middle between two plaquettes, as shown by the slightly increased apparent height of the corresponding $\mathrm{O}$ row (Fig. 3c). In the following, we name such an $\mathrm{Fe}$ atom as an interstitial Fe atom (IFA). The $\mathrm{d} I / \mathrm{d} V$ spectra taken on the IFAs show one or two tiny replicas of the coherence peaks outside the gap (Supplementary Fig. 2) most probably due to spin excitations $^{29}$, indicating that the IFAs have a residual magnetic moment. Using this technique, we assembled adatoms with one, two and three IFAs in close proximity as shown by the white arrows in the STM images (Fig. 3e-h). The pictograms of various assemblies are shown in Fig. $3 \mathrm{i}$. The $\mathrm{d} I / \mathrm{d} V$ spectra on the adatoms of each of these assemblies (Fig. 3i, in order to remove the effect of the superconducting tip, their numerical deconvolution extracted as described in the Methods section is illustrated in Fig. 3j) reveal that, with increasing number of IFAs, the most intense YSR state at positive bias voltage shifts from the gap edge towards the Fermi energy $(\mathrm{A}-\mathrm{C})$ and finally appears on the negative bias voltage side (D). This observation of a shift in the binding energy of the YSR state is consistent with a decreasing effective Kondo coupling of the $\mathrm{Fe}$ adatom with the substrate mediated through the increasing number of IFAs ${ }^{22,23}$. However, since the spectra indicate a nonzero magnetic moment of the IFA (Supplementary Fig. 2), the situation is more complex, as the $\mathrm{Fe}$ adatom is additionally exchange coupled to the IFA. Both, strong ferromagnetic (FM) and antiferromagnetic (AFM) exchange interactions, are known to renormalize the effective Kondo coupling ${ }^{30-34}$. Additionally, for the FM case, the $\mathrm{d} I / \mathrm{d} V$ spectra are expected to show multiple YSR peaks while, for the AFM case, only one pair of shifted peaks is expected $^{20,21,35}$. Indeed, a careful analysis of the spectrum corresponding to adatom B (Supplementary Fig. 3) shows additional small peaks, indicating a small FM interaction between the adatom and the IFA for this particular case. Since the surface is theoretically shown to be metallic ${ }^{36}$, we expect an RKKY type of interaction playing a significant role in the coupling between the IFA and the Fe adatom. However, we cannot rule out the possibility of a superexchange mechanism mediated through the oxygen atoms, which are bridging the adatom and the IFA. A detailed theoretical treatment of the exchange mechanism is beyond the scope of the current paper.

Spin coupling in pairs of Fe adatoms using one IFA. Motivated by the results described above, we investigate the coupling of a pair of adatoms with one IFA in the center using the technique of the switching of the adatoms between their YSR-on and -off states (Fig. 4). When both adatoms are in the YSR-on state (middle panel in Fig. 4a), they exhibit a YSR state at a distinct energy similar to that of the type B structure in Fig. 3f, which is clearly different from that of the Fe pair without IFA (c.f. Fig. 1e). However, the bound state energy positions of the two adatoms are not exactly the same showing an asymmetric coupling of both adatoms in the pair to the IFA. The asymmetry could possibly result from either a slightly asymmetric position of the IFA between the two adatoms or an intrinsic variance in the structure of the plaquettes of $(3 \times 3)$ Ta atoms (Fig. 1a), which can result in a modified coupling of the IFA with the adatoms on top. When one of the two adatoms in the pair is switched into the YSR-off state (upper and lower panels in Fig. 4a, b), the YSR state of the
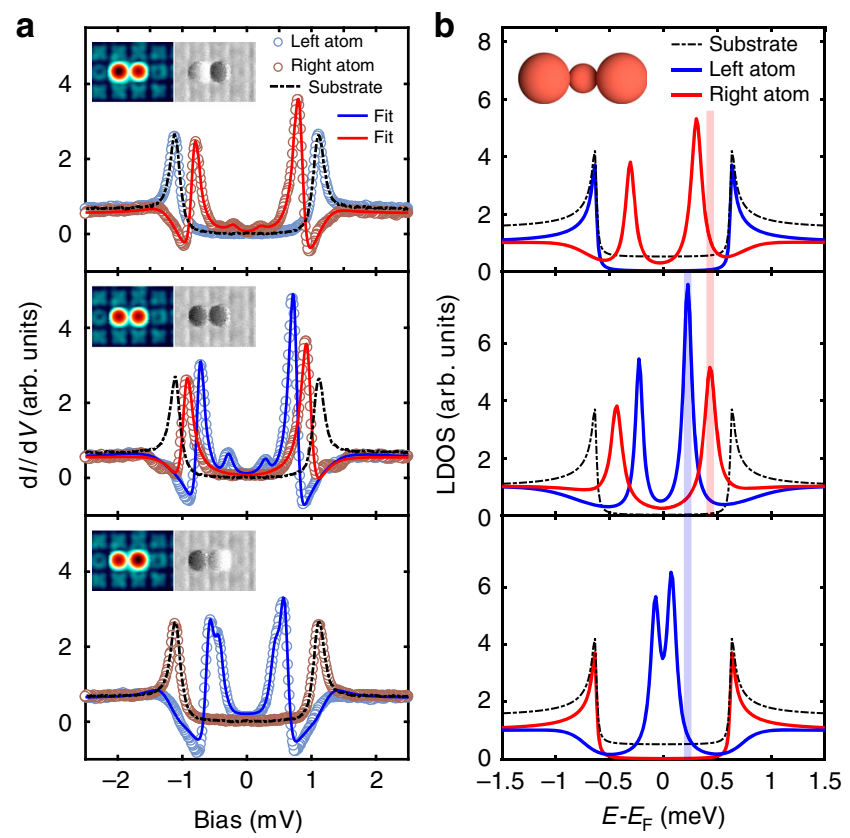

Fig. 4 Spectroscopy on a pair with one IFA in the center. a $d / / d V$ spectra taken with a superconducting tip on the two adatoms plotted together with substrate spectrum for the three cases: (1) top panel: left atom in YSR-off state and right atom in YSR-on state, (2) middle panel: both atoms in YSRon state, (3) bottom panel: left atom in YSR-on state and right atom in the YSR-off state. The solid curves in each panel represent a fit using the numerical deconvolution. Insets in each panel are the corresponding STM and $d l / d V$ images. $\mathbf{b}$ The numerically deconvoluted LDOS corresponding to the fits in each panel in $\mathbf{a}$. The black dashed curve corresponding to the substrate DOS is shown in each panel as a reference. Vertical lines are a guide to the eye showing shifts in the YSR state energy from the case (2) to the cases (1), (3). The pictogram in the upper panel shows the position of the Fe atoms in the assembly. Substrate DOSs in upper and lower panels are shifted vertically for clarity. Stabilization parameters for STS: $V_{\text {stab }}=2.5$ $\mathrm{mV}, I_{\text {stab }}=100 \mathrm{pA}, V_{\text {mod }}=20 \mu \mathrm{V}$ and scan parameters: $V=50 \mathrm{mV}, I=$ $100 \mathrm{pA}, V_{\text {mod }}=3 \mathrm{mV}$ 
remaining YSR-on adatom shifts towards the Fermi level. Note, that this result is strikingly different from that of the pairs and chains without IFAs (Fig. 2e-g), where no changes in the spectra were observed when switching one of the atoms into the YSR-off state. This clearly shows that the interaction between two neighboring adatoms can be increased via the IFA (see additional spectra on a pair with an IFA in Supplementary Fig. 4).

Chains of Fe adatoms with interstitial Fe atoms. We consequently extend the scheme described above to the formation of a chain of 13 adatoms interacting via IFAs. To this end, we initially created a chain of IFAs (Fig. 5a) followed by manipulating a chain of adatoms on top (Fig. 5b). The $\mathrm{d} I / \mathrm{d} V$ spectra of all adatoms of this chain (solid lines of Fig. 5d) show the strongest YSR state well inside the gap, and a strong variation of the YSR binding energies along the chain. To check the coupling along the chain, the sixth Fe atom from the top was switched from the YSR-on to the YSRoff state (Fig. $5 \mathrm{c}$ ), and the according spectra are shown by dashed lines in Fig. 5d. The switching has a most prominent effect on the fifth adatom from the top (Fig. 5e, f). The deconvoluted spectra (Fig. 5f) clearly show that at least four prominent peaks are needed in order to correctly capture the details of the spectra in Fig. 5e (see Supplementary Fig. 5). This implies that there is a considerable splitting of the YSR state, which is strongly indicating FM couplings ${ }^{16,21,35}$ between the adatoms in the chain. From the vertical green lines in Fig. $5 \mathrm{f}$ it is evident that, when the nearest atom is switched from YSR-on to YSR-off state, the separation between the two peaks due to the splitting of YSR states is reduced. This is consistent with a reduction of the spin coupling between the two neighbors by switching one of them into a non-magnetic state. Similar measurements on the sixth atom (see Supplementary Fig. 6) also show the splitting of the YSR state. However in this case, when the nearest neighbor is switched into the YSR-off state, we do not see a change in the splitting within our energy resolution, but only a strong variation of the intensities of the peaks. While these results evidence an exchange interaction between the adatoms in the chain, we cannot rule out that switching the atom from YSR-on to YSR-off may imply a small structural change in the nearest neighbors of the switched atom, which might cause additional shifts in the YSR states $^{37}$. Figure $5 \mathrm{~d}$, furthermore reveals that the switching of the sixth atom has a prominent influence only on the fifth atom, but not on the seventh atom, which is suggestive of an asymmetric coupling along the chain. Finally, similar switching experiments performed on all the other atoms of the chain (not shown) reveal distinct changes in the spectral features only in the nearest neighbors of the switched adatom, while the spectra away from this atom show little changes. Accordingly, we conclude that the IFAs indeed induce a short-range exchange coupling between the Fe adatoms in the chain. Note, that the short-range couplings in combination with the inherent asymmetry in the coupling between two adatoms in a pair hamper the formation of a coherent YSR band along the chain. We accordingly do not observe any signature of zero bias peaks at the chain ends in the present study. We, however, anticipate that stronger and longrange couplings might be possible by positioning additional IFAs aside the chain which could result in the formation of Majorana end modes. Therefore, our system provides an ideal playground for the tuning of various couplings via IFAs and additionally is a promising system to explore novel physics of spin-related phenomena in superconductor-spin chain hybrids.

\section{Discussion}

In summary, we showed that the system of $\mathrm{Fe}$ adatoms on the $\mathrm{Ta}$ $(100)-(3 \times 3)$ O surface provides a promising playground for crafting magnetic chains of various length coupled to an s-wave superconductor, and tuning the different couplings within the system by IFAs. We demonstrated a strengthening of the magnetic interactions along the chain via the IFAs and a manipulation of the binding energy of the YSR state. This method can be

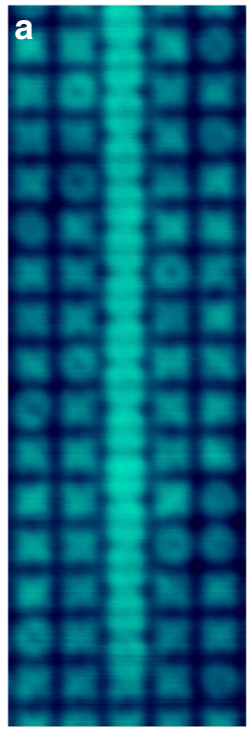

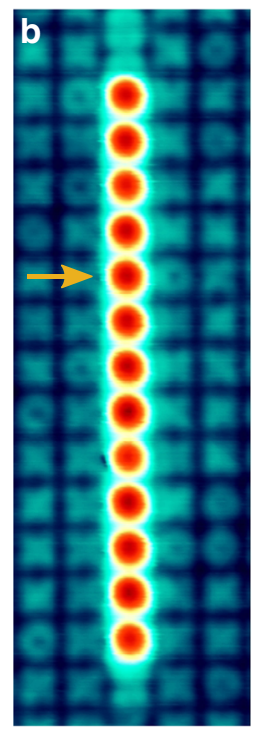

$0 \AA$

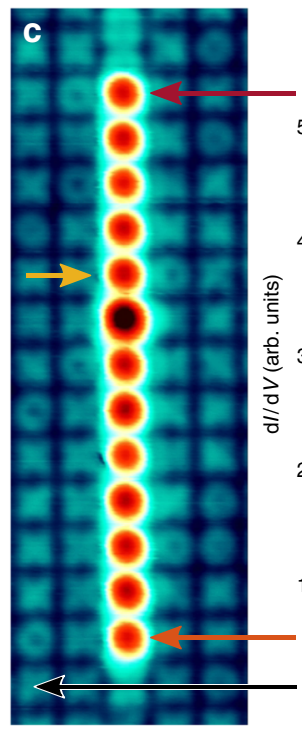

$4 \AA$
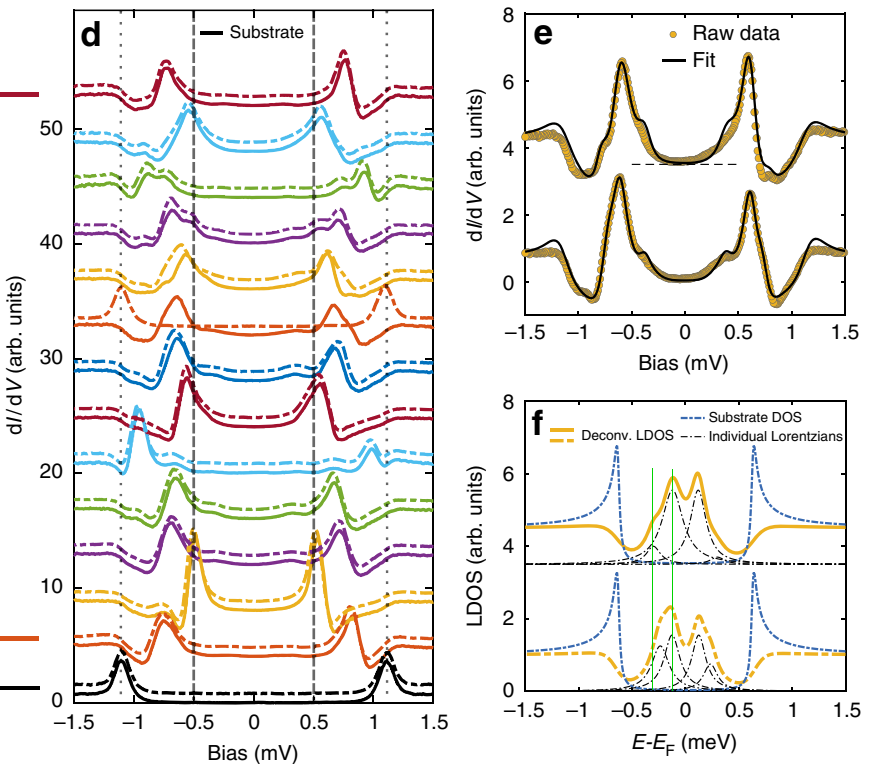

Fig. 5 Spectroscopy on a chain of 13 atoms. a STM image of manipulated IFAs before the construction of the chain. $\mathbf{b}$, c STM images of the chain with the sixth atom from the top in YSR-on and YSR-off state, respectively. The remaining atoms are in the YSR-on state for the two cases. $\mathbf{d}$ dl/dV spectra taken with a superconducting tip on each adatom of the chain corresponding to $\mathbf{b}$ (continuous curves) and $\mathbf{c}$ (dashed curves). Black curves at the bottom represent substrate spectra. The spectra are shifted vertically for clarity. The dashed (dotted) vertical lines indicate the sample Fermi level (sample coherence peak). e $d l / d V$ spectra on the fifth atom from the top (same as orange curves in $\mathbf{d}$, together with the fits from numerical deconvolution of the spectra in two different situations corresponding to $\mathbf{b}$ (top curves) and $\mathbf{c}$ (bottom curves). $\mathbf{f}$ The numerically deconvoluted LDOS corresponding to the fits in $\mathbf{e}$. The substrate DOS is plotted as a reference. Vertical green lines in $\mathbf{f}$ are guides to the eye showing the peak position of the YSR states in the upper most curve. Stabilization parameters for STS: $V_{\text {stab }}=2.5 \mathrm{mV}, I_{\text {stab }}=100 \mathrm{pA}, V_{\text {mod }}=20 \mu \mathrm{V}$ and scan parameters: $V=50 \mathrm{mV}, I=50 \mathrm{pA}$ 
extended to more complex systems as, e.g., networks of coupled chains $^{38}$. Our work is thus an important step towards the controlled realization and manipulation of topological superconductivity and Majorana end modes.

\section{Methods}

Experimental methods. All STM and scanning tunneling spectroscopy measurements were carried out in a custom built SPECS STM under ultra-high vacuum conditions and at the base temperature of $1.1 \mathrm{~K}$ using an electrochemically etched bulk Cr-tip ${ }^{39}$ coated with tantalum ${ }^{22}$. Details of the surface cleaning as well as $\mathrm{Fe}$ atom deposition can be found elsewhere ${ }^{22}$.

Prior to the atom manipulation to create various arrays of magnetic adatoms, the surface was cleaned (Fig. 1a) by picking up all the adatoms using vertical manipulation. To this end, the tip was initially held on the atom with typical stabilization parameters of $V=50 \mathrm{mV}$ and $I=50 \mathrm{pA}$. With feedback off, a typical bias voltage pulse of $\sim+1 \mathrm{~V}$ was applied in order to transfer the atom to the tip. For the transfer of $\mathrm{Fe}$ atoms from the tip to the center of the plaquettes or in-between two plaquettes the feedback was switched off and the tip was lowered by 300-600 $\mathrm{pm}$ and a negative bias voltage pulse of $0.3-1 \mathrm{~V}$ was applied in discrete steps until an Fe atom dropped, as seen in both the $z$ position of the tip and the image acquired afterwards. We did not observe YSR states on the Fe atoms adsorbed on the circular plaquettes. Therefore, we created all the atomic arrays by adatoms positioned on the cross-shaped plaquettes. Once the desired atomic arrays were created, the tip was mechanically dipped into the Ta surface in order to coat the tip apex with a superconducting Ta cluster.

Differential conductance spectra $(\mathrm{d} I / \mathrm{d} V)$ were measured using a standard lockin technique ${ }^{40}$ after stabilizing the tip at $V_{\text {stab }}$ and $I_{\text {stab }}$ with a typical modulation voltage of $V_{\text {mod }}=20 \mu \mathrm{V}(f=827 \mathrm{~Hz})$.

Numerical deconvolution of the spectra. For the deconvolution of the spectra measured with a superconducting tip, initially the tip density of states $N_{\mathrm{t}}$ is obtained by numerically fitting the substrate spectrum to the expression for the lock-in detected tunneling spectrum of a superconductor-insulator-superconductor junction, given by ${ }^{40}$

$$
\frac{\mathrm{d} I}{\mathrm{~d} V}(V) \propto \int_{-\pi / 2}^{\pi / 2} \sin \alpha \cdot I\left(V+\sqrt{2} V_{\bmod } \sin \alpha, T\right) \mathrm{d} \alpha .
$$

Here, $V_{\text {mod }}$ is the modulation voltage used for the lock-in measurements and

$$
I(V, T) \propto \int_{-\infty}^{\infty} N_{\mathrm{t}}(E) \cdot N_{\mathrm{s}}(E+V) \cdot[f(E)-f(E+V)] \cdot \mathrm{d} E,
$$

Where, $f(E)$ is the Fermi function and $N_{\mathrm{t}(\mathrm{s})}$ is the BCS-Dynes density of states of the tip (sample) given by

$$
N(E, \Gamma)=N_{\mathrm{n}}\left(E_{\mathrm{F}}\right) \cdot \Re\left[\frac{E+i \Gamma}{\sqrt{(E+i \Gamma)^{2}-\Delta^{2}}}\right] .
$$

We get the best fitting parameters for the tip with $\Delta_{\mathrm{t}}=0.5 \mathrm{meV}$ and $\Gamma_{\mathrm{t}}=0.04 \mathrm{meV}$. Once the tip is characterized, we model the density of states for the YSR states as a sum of a gap and $n$ Lorentzian peaks given by ${ }^{23}$,

$$
N_{\mathrm{s}}=\frac{1}{e^{\frac{\Delta_{\mathrm{s}}-|E|}{\delta_{\mathrm{s}}}}+1}+\sum_{i=1}^{n} \frac{A_{i}}{1+\left(\frac{E-E_{i}}{\gamma_{i}}\right)^{2}}
$$

Here, $\Delta_{\mathrm{s}}, \delta_{\mathrm{s}}, A_{i}, E_{i}$ and $\gamma_{i}$ are the free parameters which are determined using nonlinear least square fitting.

Data availability. The authors declare that all the relevant data are included in the paper and its Supplementary Information files. Additional data are available from the corresponding author upon request.

Received: 1 February 2018 Accepted: 18 July 2018

Published online: 14 August 2018

\section{References}

1. Majorana, E. Symmetrical theory of electrons and positrons. Nuovo Cim. 14, 171-184 (1937).

2. Elliott, S. R. \& Franz, M. Colloquium: Majorana fermions in nuclear, particle, and solid-state physics. Rev. Mod. Phys. 87, 137-163 (2015).
3. Alicea, J. New directions in the pursuit of Majorana fermions in solid state systems. Rep. Prog. Phys. 75, 076501 (2012).

4. Alicea, J., Oreg, Y., Refael, G., Von Oppen, F. \& Fisher, M. P. A. Non-Abelian statistics and topological quantum information processing in $1 \mathrm{D}$ wire networks. Nat. Phys. 7, 412-417 (2011).

5. Nayak, C., Simon, S. H., Stern, A., Freedman, M. \& Das Sarma, S. NonAbelian anyons and topological quantum computation. Rev. Mod. Phys. 80, 1083-1159 (2008)

6. Nadj-Perge, S., Drozdov, I. K., Bernevig, B. A. \& Yazdani, A. Proposal for realizing Majorana fermions in chains of magnetic atoms on a superconductor. Phys. Rev. B 88, 020407 (2013).

7. Klinovaja, J., Stano, P., Yazdani, A. \& Loss, D. Topological superconductivity and Majorana fermions in RKKY systems. Phys. Rev. Lett. 111, 186805 (2013)

8. Pientka, F., Glazman, L. I. \& von Oppen, F. Topological superconducting phase in helical Shiba chains. Phys. Rev. B 88, 155420 (2013).

9. Kim, Y., Cheng, M., Bauer, B., Lutchyn, R. M. \& Das Sarma, S. Helical order in one-dimensional magnetic atom chains and possible emergence of Majorana bound states. Phys. Rev. B 90, 060401 (2014).

10. Nadj-Perge, S. et al. Observation of Majorana fermions in ferromagnetic atomic chains on a superconductor. Science 346, 602-607 (2014).

11. Feldman, B. E. et al. High-resolution studies of the Majorana atomic chain platform. Nat. Phys. 13, 286-291 (2016).

12. Jeon, S. et al. Distinguishing a Majorana zero mode using spin-resolved measurements. Science 358, 772-776 (2017).

13. Ruby, M., Heinrich, B. W., Peng, Y., von Oppen, F. \& Franke, K. J. Exploring a proximity-coupled Co chain on $\mathrm{Pb}(110)$ as a possible Majorana platform. Nano. Lett. 17, 4473-4477 (2017).

14. Ruby, M. et al. End states and subgap structure in proximity-coupled chains of magnetic adatoms. Phys. Rev. Lett. 115, 197204 (2015).

15. Kim, H. et al. Toward tailoring Majorana bound states in artificially constructed magnetic atom chains on elemental superconductors. Sci. Adv. 4, eaar5251 (2018)

16. Yu, L. Bound state in superconductors with paramagnetic impurities. Acta Phys. Sin. 21, 75 (1965).

17. Shiba, H. Classical spins in superconductors. Prog. Theor. Phys. 40, 435-451 (1968).

18. Rusinov, A. I. On the theory of gapless superconductivity in alloys containing paramagnetic impurities. Sov. J. Exp. Theor. Phys. 29, 1101 (1969).

19. žitko, R., Bodensiek, O. \& Pruschke, T. Effects of magnetic anisotropy on the subgap excitations induced by quantum impurities in a superconducting host. Phys. Rev. B 83, 054512 (2011).

20. Ji, S.-H. et al. High-resolution scanning tunneling spectroscopy of magnetic impurity induced bound states in the superconducting gap of $\mathrm{Pb}$ thin films. Phys. Rev. Lett. 100, 226801 (2008).

21. Flatté, M. E. \& Reynolds, D. E. Local spectrum of a superconductor as a probe of interactions between magnetic impurities. Phys. Rev. B 61, 14810-14814 (2000).

22. Cornils, L. et al. Spin-resolved spectroscopy of the Yu-Shiba-Rusinov states of individual atoms. Phys. Rev. Lett. 119, 197002 (2017).

23. Franke, K. J., Schulze, G. \& Pascual, J. I. Competition of superconducting phenomena and kondo screening at the nanoscale. Science 332, 940-944 (2011).

24. Sahlberg, I., Westström, A., Pöyhönen, K. \& Ojanen, T. Engineering onedimensional topological phases on p-wave superconductors. Phys. Rev. B 95, 184512 (2017).

25. Stroscio, J. \& Eigler, D. Atomic and molecular manipulation with the scanning tunneling microscope. Science 254, 1319-1326 (1991).

26. Hla, S. W. Atom-by-atom assembly. Rep. Prog. Phys. 77, 056502 (2014).

27. Zhang, J. L. et al. Towards single molecule switches. Chem. Soc. Rev. 44, 2998-3022 (2015)

28. Lee, H. J. Single-bond formation and characterization with a scanning tunneling microscope. Science 286, 1719-1722 (1999).

29. Heinrich, B. W., Braun, L., Pascual, J. I. \& Franke, K. J. Protection of excited spin states by a superconducting energy gap. Nat. Phys. 9, 765-768 (2013).

30. Anders, F. B., Qin, Q. \& Grewe, N. Competing local exchange mechanisms: a validity test of a simple scaling hypothesis. J. Phys. Condens. Matter 4, 7229-7246 (1992).

31. Boucaï, E., Lecoanet, B., Pilon, J., Tholence, J. L. \& Tournier, R. Interaction effects between nearly magnetic cobalt impurities in gold. Phys. Rev. B 3, 3834-3846 (1971).

32. Heersche, H. B. et al. Kondo effect in the presence of magnetic impurities. Phys. Rev. Lett. 96, 017205 (2006).

33. Chen, W., Jamneala, T., Madhavan, V. \& Crommie, M. F. Disappearance of the Kondo resonance for atomically fabricated cobalt dimers. Phys. Rev. B 60, R8529(R) (1999).

34. Wahl, P. et al. Exchange interaction between single magnetic adatoms. Phys Rev. Lett. 98, 056601 (2007)

35. Choi, D.-J. et al. Influence of magnetic ordering between $\mathrm{Cr}$ adatoms on the Yu-Shiba-Rusinov states of the $\beta$-Bi2Pd superconductor. Phys. Rev. Lett. 120, 167001 (2018). 
36. Guo, Y. et al. Tantalum surface oxidation: bond relaxation, energy entrapment, and electron polarization. Appl. Surf. Sci. 396, 177-184 (2017)

37. Bryant, B., Spinelli, A., Wagenaar, J. J. T., Gerrits, M. \& Otte, A. F. Local control of single atom magnetocrystalline anisotropy. Phys. Rev. Lett. 111, 127203 (2013)

38. Björnson, K. \& Black-Schaffer, A. M. Majorana fermions at odd junctions in a wire network of ferromagnetic impurities. Phys. Rev. B 94, 100501 (2016).

39. Manna, S. et al. Interfacial superconductivity in a bi-collinear antiferromagnetically ordered FeTe monolayer on a topological insulator. Nat. Commun. 8, 14074 (2017).

40. Löptien, P., Zhou, L., Khajetoorians, A. A., Wiebe, J. \& Wiesendanger, R. Superconductivity of lanthanum revisited: enhanced critical temperature in the clean limit. J. Phys. Condens. Matter 26, 425703 (2014).

\section{Acknowledgements}

The authors thank Maria Valentyuk, Roberto Mozara, Alexander I. Lichtenstein, Saurabh Pradhan and Jonas Fransson for fruitful discussions, and Dörte Langemann for help with the manipulation. This work has primarily been supported by the ERC Advanced Grant ASTONISH (No. 338802). L.C. and J.W. acknowledge support through the DFG priority programme SPP1666 (grant no. WI 3097/2).

\section{Author contributions}

All authors conceived and designed the experiments. A.K. and L.C. performed the experiments. A.K. analyzed the data. A.K. and J.W. wrote the manuscript. All authors discussed the results and commented on the manuscript.

\section{Additional information}

Supplementary Information accompanies this paper at https://doi.org/10.1038/s41467018-05701-8.

Competing interests: The authors declare no competing interests.

Reprints and permission information is available online at http://npg.nature.com/ reprintsandpermissions/

Publisher's note: Springer Nature remains neutral with regard to jurisdictional claims in published maps and institutional affiliations.

(c) (i) Open Access This article is licensed under a Creative Commons Attribution 4.0 International License, which permits use, sharing, adaptation, distribution and reproduction in any medium or format, as long as you give appropriate credit to the original author(s) and the source, provide a link to the Creative Commons license, and indicate if changes were made. The images or other third party material in this article are included in the article's Creative Commons license, unless indicated otherwise in a credit line to the material. If material is not included in the article's Creative Commons license and your intended use is not permitted by statutory regulation or exceeds the permitted use, you will need to obtain permission directly from the copyright holder. To view a copy of this license, visit http://creativecommons.org/ licenses/by/4.0/.

(c) The Author(s) 2018 